\title{
Adiabatic Radiofrequency Pulse Forms in Biomedical Nuclear Magnetic Resonance
}

\author{
DAVID G. NORRIS* \\ Max-Planck-Institute of Cognitive Neuroscience, Stephanstr. 1a, D-04103 Leipzig, Germany
}

\begin{abstract}
Adiabatic radio frequency (RF) pulses are in widespread use in biomedical magnetic resonance imaging and spectroscopy. The primary advantage of adiabatic pulses is that provided the condition for adiabaticity is satisfied they can be made insensitive to inhomogeneities in the RF field. In this pedagogical article the principles of adiabatic fast passage (AFP) are explained, and the use of AFP to invert both stationary and flowing spin systems is examined. The hyperbolic secant pulse is presented as a pulse capable of performing slice selective adiabatic inversion. Lower power alternatives to this pulse are described, and the principle of offset independent constant adiabaticity is elucidated. Instantaneous reversal of the orientation of the effective RF field as a means of producing excitation and refocusing pulses is presented, as are methods of obtaining slice selective excitation with these pulses. (c) 2002 Wiley Periodicals, Inc. Concepts Magn Reson 14: 89-101 2002
\end{abstract}

KEY WORDS: adiabatic RF; adiabatic fast passage; hyperbolic secant pulse

\section{INTRODUCTION}

Radio frequency (RF) pulse forms that rely on the principle of adiabatic fast passage (AFP) (1) have found widespread utility in biomedical nuclear magnetic resonance (NMR). Pulses based on AFP cannot at present be designed using the formalism developed by Shinnar and Le Roux (2-8) and are

Received 18 June 2001; revised 23 October 2001; accepted 2 November 2001.

${ }^{*}$ Present address: FC Donders Centre for Cognitive Neuroimaging, Trigon 181, P.O. Box 9101, NL 6500 HB Nijmegen, The Netherlands; e-mail: David.Norris@FCDonders.kun.nl.

Concepts in Magnetic Resonance, Vol. 14(2) 89-101 (2002)

DOI 10.1002/cmr.10007

(C) 2002 Wiley Periodicals, Inc. best understood by recourse to a mixture of vector sweep-diagrams and mathematical arguments. The attraction of AFP pulses is their insensitivity, over a broad range, to inhomogeneities in the $\mathbf{B}_{1}$-field. This is particularly important in biomedical applications because of the desire to examine relatively large volumes, up to the size of the human torso, or in some situations to employ surface coils both for RF transmission and signal reception.

The purpose of this article is educational and is aimed at acquainting the reader with the principles of adiabatic fast passage, and the adiabatic RF pulses and some of the applications which are encountered in biomedical NMR. It is not intended to provide an all encompassing review. For a review of the use of adiabatic pulses in biomedical NMR the reader is referred to (9). 


\section{ADIABATIC FAST PASSAGE}

The classical understanding of AFP is based on a precession argument in the rotating frame of reference. It is well known that in the frame rotating at the Larmor frequency $\omega_{0}$ the magnetisation vector (M) will precess about the effective $\mathbf{B}_{1}$-field $\mathbf{B}_{1 e}$. For a pulse having a frequency which differs from $\omega_{0}$ by $\Delta \omega$ this effective field is given by

$$
\mathbf{B}_{1 e}=\mathbf{B}_{1}+\frac{\Delta \omega}{\gamma} \mathbf{k}
$$

where $\mathbf{B}_{1}$ is a vector in the transverse plane of the rotating coordinate system and $\mathbf{k}$ is a unit vector along the $z^{\prime}$-axis. In this article trajectories of $\mathbf{B}_{1 e}$ will generally be considered in a frame of reference rotating with the instantaneous frequency of the RF pulse. In this frame sweeps are most easily visualized; occasionally a second frame of reference will be used, denoted by $\left(x^{\prime \prime}, y^{\prime \prime}, z^{\prime \prime}\right)$ in which the $z^{\prime \prime}$-axis rotates in parallel with the $\mathbf{B}_{1 e}$-field. For convenience the quantity $\frac{\Delta \omega}{\gamma}$ will often be reffered to as $\mathbf{B}_{z}$. The orientation of these vectors is indicated in Fig. 1. In a standard RF pulse the frequency is held constant, and the precession about $\mathbf{B}_{1 e}$ is used to change the orientation of $\mathbf{M}$, so of necessity the angle between $\mathbf{B}_{1 e}$ and $\mathbf{M}$ is large. In AFP the orientation of $\mathbf{B}_{1 e}$ is changed



Figure 1 Sketch showing the effective magnetic field $\mathbf{B}_{1 e}$ in the frame of reference rotating with the instantaneous frequency of the RF pulse, and its two component vectors $\mathbf{B}_{1}$ and $\mathbf{B}_{z}$, where $\mathbf{B}_{z}=\frac{\Delta \omega}{\gamma}$. The angle $\theta$ is defined as that subtended by $\mathbf{B}_{1 e}$ and $\mathbf{B}_{1}$. The magnetization $\mathbf{M}$ is shown as a shaded cone precessing about $\mathbf{B}_{1 e}$. The adiabatic sweep is described by a rotation about the vector $\Omega$ which is shown as lying in the $\left(x^{\prime}, y^{\prime}\right)$-plane. The $z^{\prime \prime}$-axis of the frame of reference that rotates with $\mathbf{B}_{1 e}$ is also shown. The $x^{\prime \prime}$ - and $y^{\prime \prime}$-axes would initially be aligned with the $x^{\prime}$ - and $y^{\prime}$-axes and rotate with $\mathbf{B}_{1 e}$ but have been omitted for clarity. in such a way that the angle subtended by $\mathbf{M}$ and $\mathbf{B}_{1 e}$ remains constant throughout the pulse, and $\mathbf{M}$ hence moves with $\mathbf{B}_{1 e}$. The term "adiabatic" arises because the change in the orientation of $\mathbf{B}_{1 e}$ must be sufficiently slow that $\mathbf{M}$ is able to follow it. However, the total duration of the pulse must be shorter than any relaxation processes and hence the term "fast passage." Following Abragam (1) we can consider the motion of $\mathbf{M}$ in the reference frame $\left(x^{\prime \prime}, y^{\prime \prime}, z^{\prime \prime}\right)$. The motion of $\mathbf{B}_{1 e}$ can be described by a rotation about a vector $\Omega$ perpendicular to the $z^{\prime}$-axis as is shown in Fig. 1 . The equation of motion of $\mathbf{M}$ in this reference frame is given by the well known equation for transformation to a rotating coordinate system by

$$
\frac{\partial \mathbf{M}}{\partial t}=\gamma \mathbf{M} \times\left(\mathbf{B}_{1 e}+\frac{\Omega}{\gamma}\right) .
$$

If

$$
|\Omega| \ll\left|\gamma \mathbf{B}_{1 e}\right|
$$

and remembering that in this frame $\mathbf{B}_{1 e}$ is aligned parallel to the $z^{\prime \prime}$-axis, then we can write

$$
\frac{\partial M_{x, y}}{\partial t} \cong \gamma\left|\mathbf{B}_{1 e}\right| M_{x, y} .
$$

The solution of Eq. [4] represents a rotation at constant magnitude, and hence a constant angle of precession of $\mathbf{M}$ about $\mathbf{B}_{1 e}$. Equation [3] represents the most fundamental condition for adiabaticity. If we now include the consideration that an adiabatic pulse must be faster than any relaxation processes then we have

$$
\frac{1}{T_{2}}, \frac{1}{T_{2}^{*}} \ll|\Omega| \ll\left|\gamma \mathbf{B}_{1 e}\right| .
$$

Classically only $T_{2}$ relaxation is considered, which sets an absolute physical limit for the pulse duration which is independent of experimental conditions. However, particularly for in vivo applications, $T_{2}^{*}$ may be more critical.

If we consider a rotation of $\mathbf{B}_{1 e}$ from the positive to the negative $z^{\prime}$-axis, i.e., an adiabatic inversion, in which the amplitude of the $\mathbf{B}_{1}$ field is held constant and the sweep is effected solely by modulation of the frequency offset then

$$
\tan \theta=\frac{\left|\mathbf{B}_{z}\right|}{\left|\mathbf{B}_{1}\right|},
$$

and as only $\mathbf{B}_{z}$ varies with time

$$
\dot{\theta} \sec ^{2} \theta=-\frac{\mid}{\dot{\mathbf{B}}_{z} \mid}\left|\mathbf{B}_{1}\right| .
$$


After some manipulation it is then possible to write.

$$
\Omega=\dot{\theta}=-\frac{\mathbf{B}_{1} \dot{\mathbf{B}}_{z}}{\mathbf{B}_{1 e}^{2}} .
$$

Insertion of the inequality given in Eq. [3] gives the result that

$$
\left|\dot{\mathbf{B}}_{z}\right| \ll\left|\frac{\gamma \mathbf{B}_{1 e}^{3}}{\mathbf{B}_{1}}\right| .
$$

This condition is most sorely tested when $\mathbf{B}_{1 e}$ passes through the transverse plane and has its minimum value of $\mathbf{B}_{1 e}=\mathbf{B}_{1}$ at which point Eq. [9] simplifies to

$$
\left|\dot{\mathbf{B}}_{z}\right| \ll \gamma \mathbf{B}_{1}^{2} .
$$

On resonance the trajectory of $\mathbf{B}_{1 e}$ will pass through the transverse plane midway throughout the sweep. In the presence of a frequency offset the passage through the transverse plane will be shifted in time dependent upon the size of the frequency offset relative to the frequency width of the sweep. The inversion will still be successful provided that this frequency width is sufficiently large that $\mathbf{B}_{1 e}$ is aligned along the $z^{\prime}$-axis both at the start and at the end of the sweep. Provided that Eq. [10] is satisfied, inversion will occur over a range of $\mathbf{B}_{1}$ values. The efficiency of the inversion will also be independent of the exact value of $\mathbf{B}_{1}$ within this range. These properties made AFP attractive for inversion in the presence of significant inhomogeneities in both the $\mathbf{B}_{0}$ and the $\mathbf{B}_{1}$ fields (10). Termination of the sweep at the halfway point will result in a saturation pulse which will be similarly insensitive to inhomogeneities in $\mathbf{B}_{1}$ as the inversion pulse. However, the efficiency of the saturation will depend on the frequency offset and hence saturation pulses will show some sensitivity to inhomogeneity in $\mathbf{B}_{0}$, as will be discussed below in the context of halfsech pulses. It is an important general point that this sensitivity to off-resonance effects makes simple AFP sweeps generally unsuitable for excitation and refocusing, and for use in combination with slice selection employing magnetic field gradients.

AFP may be achieved by three methods in practice: by holding the frequency of the RF-pulse constant and sweeping the $\mathbf{B}_{0}$-field, by sweeping the frequency of the RF-pulse, and by appropriately modulating the phase of the RF-pulse. Historically the first of these variants was of importance in the days of $\mathrm{CW}$ spectrometers as the RF was held constant and the magnetic field swept through resonance. In modern systems it is generally more convenient to modulate the phase than to directly vary the frequency: most consoles only permit the transmission of RF pulses at a fixed carrier frequency, but the phase can generally be freely programmed at the generation of the pulse.

An interesting extension of the application of AFP is for the inversion of flowing spins. A magnetic field gradient is applied parallel to the direction of the flow as shown in Fig. 2. This experiment is really an extension of the AFP implementation in which the RF is held constant and the $\mathbf{B}_{0}$-field is swept. Motion in the direction of the gradient is used to modulate the instantaneous Larmor frequency, so spins moving with a constant velocity will experience a linear sweep of the resonance frequency, as shown in Fig. 2. Typically a small transmitter coil is used to effect the inversion, for example, placed above the carotid artery in the neck. For maximum efficiency the frequency of the RF is set equal to the Larmor frequency at the center of the coil in the presence of the gradient. As in the inversion experiment described above, the $\mathbf{B}_{1}$-field is constant and so the adiabatic condition is most tested at zero offset frequency. For spins moving with a velocity component $v$ parallel to the gradient field we have

$$
\Delta \omega=\gamma G v t
$$

where for convenience the temporal origin is taken as the time at which there is zero frequency

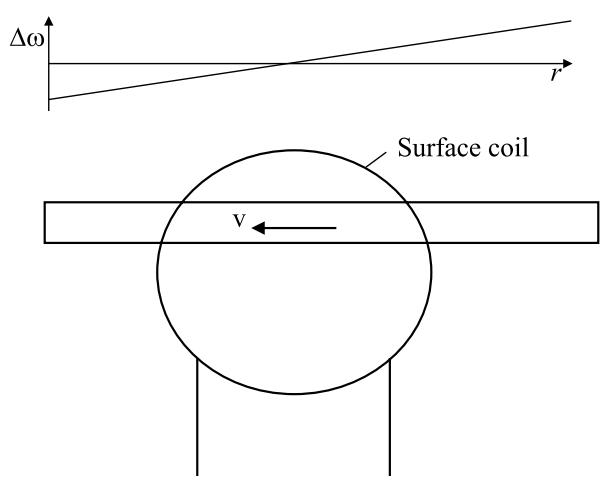

Figure 2 Flow induced adiabatic inversion. The spins flowing with velocity $\mathbf{v}$ experience a linear variation in the frequency offset due to the presence of the magnetic field gradient. The RF pulse frequency should be so adjusted that the midpoint of the inversion is roughly at the center of the surface coil. 
offset. In the general situation

$$
\Omega=\dot{\theta}
$$

where

$$
\tan \theta=\frac{G v t}{\left|\mathbf{B}_{1}\right|}
$$

for small values of $\theta, \tan \theta \cong \theta$ and hence Eq. [3] can be rewritten as

$$
\left|\frac{G v}{\mathbf{B}_{1}}\right| \ll\left|\gamma \mathbf{B}_{1}\right| .
$$

The inversion must still be faster than the appropriate relaxation time for the blood as described in Eq. [5], but there is also the additional condition that the inversion must take place while the spins are within the sensitive region of the labelling coil. This leads to the further condition that

$$
\frac{1}{T_{2}} \ll \frac{v}{d} \ll\left|\frac{G v}{\mathbf{B}_{1}}\right| \ll\left|\gamma \mathbf{B}_{1}\right|
$$

where $d$ is the diameter of the coil. Contravention of the adiabatic condition can hence occur in three situations: for slow moving spins if relaxation occurs during the transition, i.e., if the left most inequality of [15] is contravened; if the choice of gradient and $\mathbf{B}_{1}$-fields is inappropriate for the diameter of the coil, corresponding to the central inequality of Eq. [15]; and finally if the velocity is so great that the fundemental adiabatic condition is contravened which is the right hand inequality in Eq. [15].

Velocity-dependent adiabatic inversion was initially proposed for performing projective angiography in the brain (11) and was subsequently proposed as a method for performing continuous arterial spin-labelling (12-15). Two studies have also examined the efficiency of the labelling process using this technique $(16,17)$.

The approaches described above were developed during the early days of NMR. With the advent of imaging systems and the requirement for slice selective excitation it became possible to modulate the form of the $\mathbf{B}_{1}$-field. The potential advantage of such a sweep is the consideration that if a linear sweep satisfies the adiabatic condition at resonance then this condition will be exceeded by more than required for all other offsets, leading to longer pulses and/or higher RF power than necessary. One obvious modulation form is the $\sin / \cos$ form (18)

$$
\mathbf{B}_{1}(t) \propto \sin \Omega t
$$

$$
\Delta \omega \propto \cos \Omega t
$$

which ensures that $\mathbf{B}_{1 e}$ is constant throughout the pulse as of course is $\Omega$. This modulation form ensures that the adiabaticity is constant throughout the pulse, but no attempt is made to minimize the pulse duration. A natural attempt to do this is to hold both the adiabaticity and the $\mathbf{B}_{1}$-field constant and then to vary the offset frequency. This gives rise to the constant adiabaticity pulse form $(19,20)$, which may be derived as follows: The inequality given in Eq. [3] may be converted into an equation by introducing the adiabaticity factor $K$, such that

$$
|K \Omega|=\left|\gamma \mathbf{B}_{1 e}\right| .
$$

By reference to Fig. 1 we know that

$$
\mathbf{B}_{1 e}=\frac{\mathbf{B}_{1}}{\cos \theta}
$$

and so it is possible to write Eq. [18] as

$$
|\dot{\theta} \cos \theta|=\left|\frac{\gamma \mathbf{B}_{1}}{K}\right|
$$

which, if we take the temporal origin $(t=0)$ to be at the center of the sweep, may be integrated to give

$$
|\sin \theta|=\left|\frac{\gamma \mathbf{B}_{1} t}{K}\right|
$$

but

$$
\Delta \omega=\gamma \mathbf{B}_{1} \tan \theta
$$

and we can hence write

$$
\Delta \omega \propto \frac{\gamma^{2} \mathbf{B}_{1}^{2} t}{\sqrt{\left[K^{2}-\left(\gamma \mathbf{B}_{1} t\right)^{2}\right]}} .
$$

This form has the advantage that the minimum pulse duration should always be achieved, but at the start and end of the sweep effectively an infinite frequency offset is required to ensure that the $\mathbf{B}_{1 e}$-field is parallel or antiparallel to the $z^{\prime}$-axis. It is hence clear that superior performance should be expected from pulse shapes that have a tapered amplitude modulation at the start and end of the sweep. Other commonly encountered modulation forms are the constant/tan form (21), and the sech/tanh form which will be examined in detail in the next section. Some attempts have been made to optimize the performance of these sweep functions so that they can be guaranteed to function over a specified range of variation in the $\mathbf{B}_{1}$-field, using numerical approaches (22) and for some cases analytical solutions (23). 


\section{SLICE SELECTIVE INVERSION AND SATURATION}

A natural limitation of the AFP forms discussed in the previous section is that these waveforms do not have an off-resonance behavior which makes them suitable for slice selective excitation. The first pulse to be found that produces a reasonably sharp response profile for inversion was the hyperbolic secant pulse $(19,24-26)$ and its variants, which will be discussed in this section.

\section{THE HYPERBOLIC SECANT PULSE}

The hyperbolic secant or sech pulse has the complex form

$$
\widetilde{B}_{1}(t)=B_{1}^{0}(\operatorname{sech}(\beta t))^{1+i \mu}
$$

or

$$
\widetilde{B}_{1}(t)=B_{1}^{0} \operatorname{sech}(\beta t) \exp (i \mu \ln \operatorname{sech} \beta t)
$$

which represents both an amplitude and a frequency modulation and may be explicitly written as

$$
\begin{aligned}
B_{1}(t) & =B_{1}^{0} \operatorname{sech}(\beta t) \\
\Delta \omega(t) & =-\mu \beta \tanh (\beta t) .
\end{aligned}
$$

The corresponding amplitude and phase modulation is illustrated in Fig. 3. The parameter $\mu$ determines the degree of phase modulation and in combination with the parameter $\beta$, which gives the truncation level, determines the bandwidth according to

$$
\Delta \omega_{\mathrm{BW}}=2 \mu \beta .
$$

It is possible to analytically solve the Bloch equations using the time-dependent $\mathbf{B}_{1}$-field given in Eq. [24] as a driving function. The result is a broadband inversion with a fairly sharp inversion profile as shown in Fig. 4 for a range of $\mu$ values. Above a certain threshold in the $\mathbf{B}_{1}$-field strength the inversion profile is largely independent of this parameter. The analytical solution of the Bloch equation is complex (26) and shall not be given here. There are some specific insights to be gained from the analysis, one being the derivation of Eq. [27]. Further salient features as discussed in (26) are:

1. In the limit of no phase modulation $(\mu=0)$, when

$$
\mathbf{B}_{1}^{0} \approx n \gamma \beta
$$

and $n$ is an integer, the magnetization remains unaltered by the pulse. This is similar to the phenomenon of optical transparency and means that for the correct value of the $\mathbf{B}_{1}$-field strength a $2 n \pi$ rotation is achieved, independent of the frequency offset.

2. In the limit in which $\mu \rightarrow \infty$, and $\beta \rightarrow 0 \mu \beta=$ constant, an adiabatic fast passage is effectively performed.

3. Provided that $\mu \geq 2$ and $\mathbf{B}_{1}^{0} \geq \gamma \mu \beta$ the inversion is essentially independent of the strength of $\mathbf{B}_{1}^{0}$, a result given above.

Taken together these results show that the pulse has an adiabatic quality but also that the nature of the amplitude modulation is significant. Intuitively it is possible to understand the performance of the sech pulse in terms of the similarities to an adiabatic passage: as such, useful insights into the performance of the sech pulse have been gained by using sweep diagrams (27). The sweep diagram makes it possible to understand the insensitivity to the $\mathbf{B}_{1}$-field strength, as shown in Fig. 5(A). The start and end points of the trajectory are unaffected by variations in the $\mathbf{B}_{1}$-field strength; only the locus of the sweep is modified. By considering a constant offset frequency it is also possible to understand the slice selective nature of the pulse: within the slice the frequency offset is smaller than the maximum frequency offset of the pulse and so the adiabatic passage still results in a reversal of the magnetization vector. Outside the slice the frequency offset is larger and so the orientation of $\mathbf{B}_{1 e}$ cannot be reversed. These processes are illustrated in Fig. 5(B). It should be noted that outside the selected slice the magnetization is tipped out of the longitudinal axis, and subsequently returned to it. If the adiabatic condition is contravened by the pulse duration being comparable to $T_{2}$ then the longitudinal magnetization outside the selected slice will be attenuated as a result of its excursion into the transverse plane with deleterious effects on the slice profile $(28,29)$.

It is also possible to consider using half of a sech pulse in order to perform an adiabatic saturation (27). Given the sweep trajectories discussed 


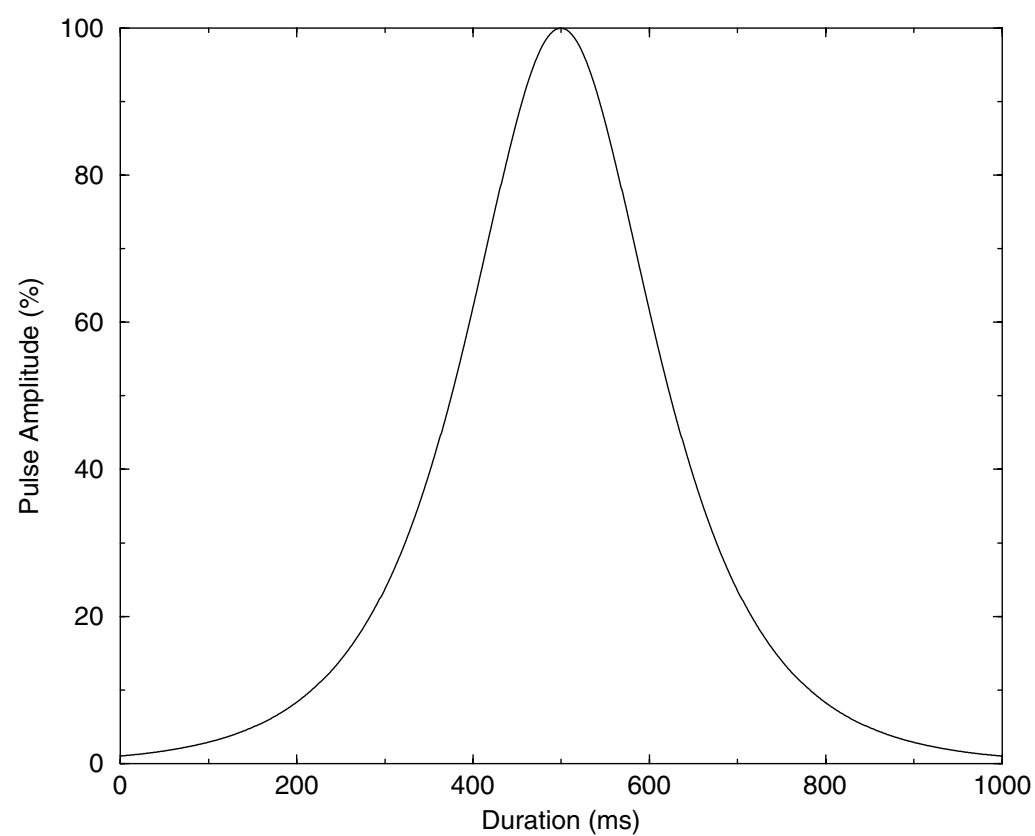

(a)

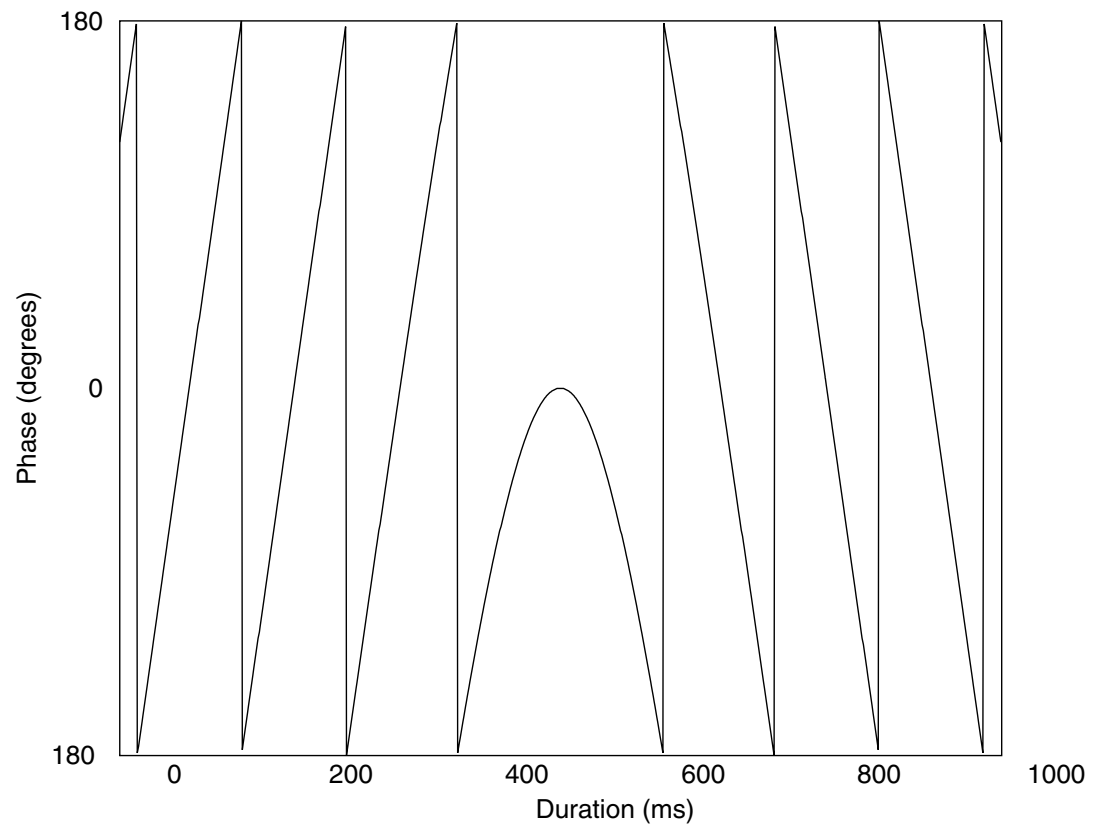

(b)

Figure 3 Amplitude (a) and phase modulation (b) for a sech pulse with $\mu=5$.

above it is hardly surprising that the excitation profile is radically different than that of the full sech pulse: the half-sech will tip the magnetization away from the longitudinal axis, without returning it there in the second half of the pulse, resulting in a far broader excitation profile. At the frequency offset $\Delta \omega=\mu \beta$ the initial frequency offset of the pulse will be exactly cancelled by the external offset (cf. Eq. [26]) and as the initial amplitude of the sech is also zero the adiabatic condition cannot be satisfied at this offset $(27,30)$. This results in a notch of the excitation profile, and the phase of the transverse magnetization is reversed in going across this singularity because on one side of the notch the magnitude of the initial frequency offset of the pulse will be greater than the external offset, causing $\mathbf{B}_{1 e}$ to be antiparallel to the initial direction of $\mathbf{M}_{0}$ whereas on the other side of the 


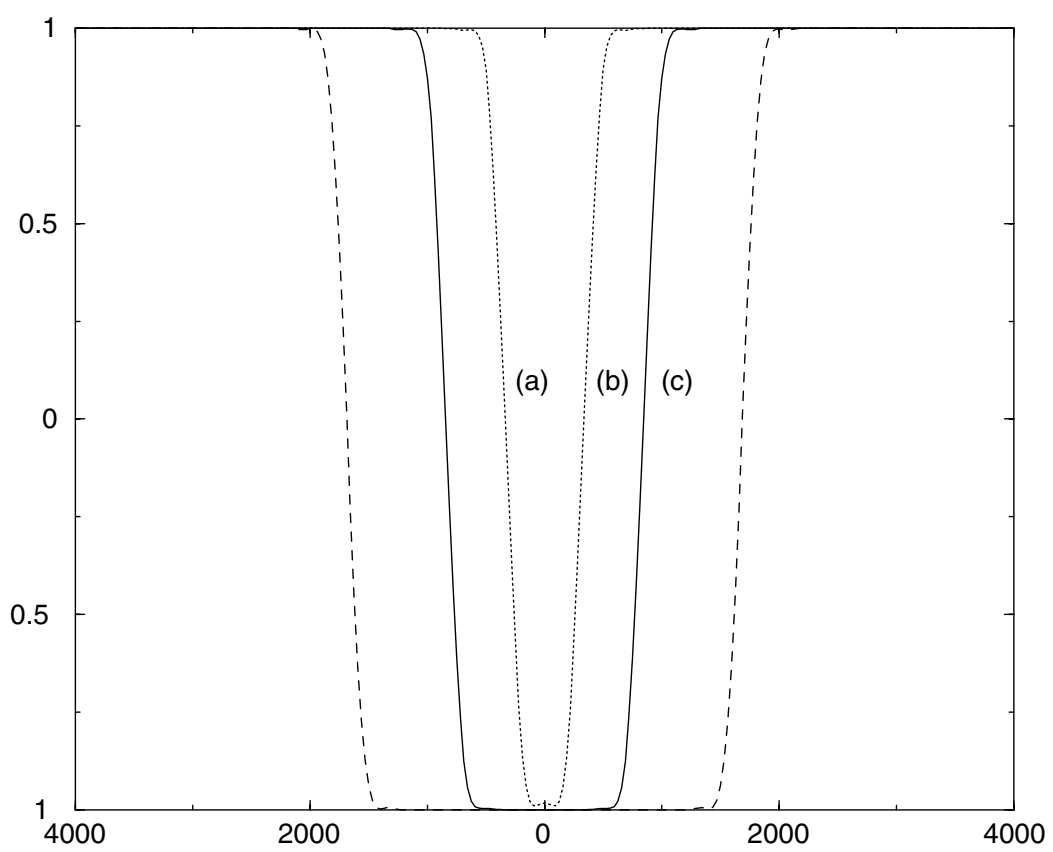

Figure 4 Inversion profiles obtained for a $10 \mathrm{~ms}$ sech pulse having the $\mu$-values of 2 (a), 5 (b), and 10 (c). The increase in both bandwidth and profile quality with increasing $\mu$-value is clearly visible.

notch the two vectors are parallel as is shown in Fig. 6 (27). The final orientation of the magnetization relative to the transverse plane is dependent only on the external frequency offset and the strength of the $\mathbf{B}_{1}$-field. This leads to the interesting result that the bandwidth is dependent only on the strength of the $\mathbf{B}_{1}$-field and not on the

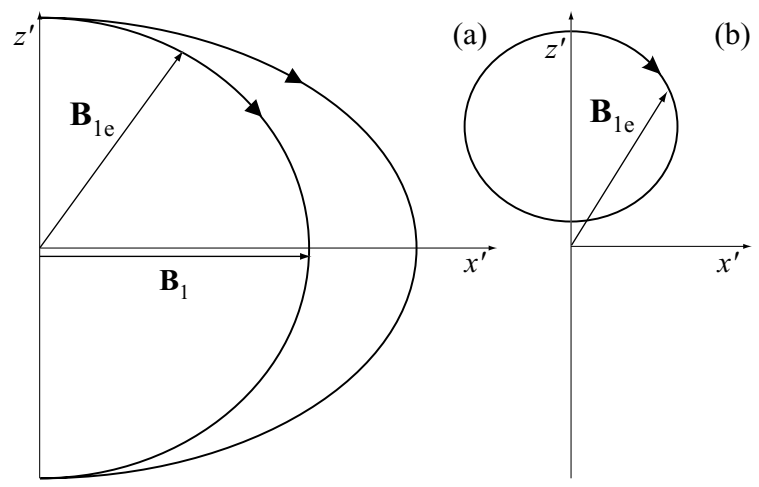

Figure 5 Sweep diagrams for slice selective adiabatic inversion. (a) shows the sweep for two different values of $\mathbf{B}_{1}$-field strength and makes the point that although the trajectories may differ significantly the same endpoint will be reached. (b) Shows how magnetization just outside the selected slice is tipped away from the $z^{\prime}$-axis, only to be returned to it at the end of the pulse. This is because the maximum frequency offset of the pulse is smaller than that due to the selection gradient. pulse duration, provided of course that the adiabatic condition is still satisfied $(27,30)$.

\section{EXTENSIONS TO THE HYPERBOLIC SECANT PULSE}

Although the hyperbolic secant pulse was very successful at producing broadband inversion, it does suffer from a relatively high peak RF power and in



Figure 6 Illustration of the phase reversal in the magnetization (M) that occurs at the frequency offset $\mu \beta$. In (a) the magnitude of the initial frequency offset of the pulse, $\Delta \omega$, is greater than $\mu \beta$ and the trajectory of $\mathbf{B}_{1 e}$ starts from the $-z^{\prime}$-axis. In (b) the converse is true. Although the final orientation of the $\mathbf{B}_{1 e}$ vectors is in both instances similar, the differing initial polarity causes the phase reversal in $\mathbf{M}$. 
some in vivo applications, such as localized spectroscopy using the ISIS technique (31) and perfusion imaging using pulsed arterial spin labelling methods, a sharper inversion profile could be desired. On the basis of the sweep diagram approach it is straightforward to conceive that any trajectory that smoothly reverses its orientation along the $z^{\prime}$-axis, by starting and finishing with a large frequency offset and a low amplitude of the $\mathbf{B}_{1}$-field, will be capable of producing a slice selective inversion. Initial attempts to improve upon the performance of the sech pulse concentrated on producing pulses which satisfy the requirement for a low initial and final amplitude of the $\mathbf{B}_{1}$-field with a constant $\mathbf{B}_{1}$-field intensity during the central period of the pulse. Using considerations of this nature Rosenfeld et al. produced a pulse made up of three types of segment which were smoothly joined (32). The performance of this pulse was subsequently optimized $(33,34)$. Using similar considerations Kupče and Freeman (35) developed the WURST pulse (an acronym ostensibly based on the formulation wideband $u$ niform rate and smooth truncation, but also invoked because the amplitude form resembles that of a sausage). This has the analytical form for the modulation of the $\mathbf{B}_{1}$-field of

$$
B_{1}(t)=B_{1}^{0}\left\{1-|\sin (\beta t)|^{n}\right\}
$$

with the frequency offset being swept as a linear function of time. The higher the value of $n$, the sharper the transition in the $\mathbf{B}_{1}$-field at the ends of the pulse, and the higher the bandwidth. The value $n=20$ was recommended in the original paper on this subject (35). In comparison to the sech pulse the WURST-20 pulse has a broader bandwidth but a somewhat shallower transition gradient. Shen (36) used a previously published transform (19) to rescale the temporal axis of known modulation functions, thus achieving a significant reduction in RF power, while maximizing the adiabaticity at resonance.

However, the approach that gives the most insight into the design of slice selective inversion pulses is that developed by Kupče and Freeman $(37,38)$ and Tannús and Garwood (39). This is an extension of the constant adiabaticity sweep described above in Eqs. [18]-[23]. The salient point in this approach is the realization that the degree of adiabaticity across the whole bandwidth of the pulse is important and not just at resonance. If we allow both the frequency offset and the amplitude of the $\mathbf{B}_{1}$-field to be time-varying, then we can rewrite Eq. [9] as

$$
K=\frac{\left(\mathbf{B}_{1}^{2}+\mathbf{B}_{z}^{2}\right)^{3 / 2}}{\dot{\mathbf{B}}_{z} \mathbf{B}_{1}-\dot{\mathbf{B}}_{1} \mathbf{B}_{z}}
$$

For spins that are off-resonance by the frequency $\Lambda, \mathbf{B}_{z}$ can be written as

$$
\mathbf{B}_{z}(t)=\frac{\Lambda}{\gamma}-F_{\omega}(t)
$$

where $F_{\omega}(t)$ represents the modulation function for $\mathbf{B}_{z}$. For all frequency offsets within the selected slice $K$ should be independent of $\Lambda$. For each $\Lambda$ within the slice there will be a time, $t_{\Lambda}$, at which the resonance condition is satisfied and $\mathbf{B}_{z}\left(t_{\Lambda}\right)$ will be zero. However, $K$ is also required to have this value for all other offsets within the slice. Equation [30] can then be rewritten as

$$
K\left(t_{\Lambda}\right) \dot{\mathbf{B}}_{z}=\mathbf{B}_{1}^{2}
$$

This equation bears a remarkable similarity to Eq. [10]. So once a suitable modulation form for the amplitude has been determined then the frequency modulation follows automatically from Eq. [32]. A key feature of this approach is that the power is distributed evenly over the whole of the inversion bandwidth. An interesting observation is that a nonadiabatic pulse with a rectangular slice profile distributes the energy equally over the bandwidth at any given time, whereas an adiabatic pulse does this sequentially (39). There is still an infinite number of pulses that satisfy Eq. [32], including, notably the sech pulse itself, but forms can be chosen which use low peak and mean $\mathbf{B}_{1}$-fields, or further optimization procedures can be sought $(40,41)$. A number of pulse forms are given in Refs. 38 and 39 ; in particular the WURST pulse in its original form does not satisfy Eq. [32], but a frequency modulation function is given in table 1 of Ref. 39 that remedies this deficit.

The realization that the inversion occurs sequentially through the slice when using the sech pulse leads to the idea that by increasing the magnetic field gradient at the start and end of the pulse, sharper edges and a reduced chemical shift artifact can be achieved relative to the original sech pulse, but at no increase in RF power (42). These pulses are collectively known as frequency offset corrected inversion pulses. A number of 
possible trajectories were presented in the original publication on this topic (42) and were subsequently improved upon by incorporating the gradient field into the principle of offset independent constant adiabaticity (9).

\section{EXCITATION AND REFOCUSING PULSES}

As discussed above, single adiabatic sweeps are incapable of performing excitation or refocusing. In this section the combination of adiabatic sweeps to achieve this end will be examined. The general principle is that by combining sweeps with different parameters the desired manipulation can be achieved and undesirable effects eliminated. Pulses which have a sufficiently well defined behavior off-resonance can be considered for slice selective schemes.

\section{Non Slice Selective Pulses}

The basic principle by which excitation or refocusing can be achieved is by utilizing instantaneous reversal of the orientation of the effective $\mathbf{B}_{1 e^{-}}$-field $(43,44)$. The principles of operation of a $90^{\circ}(44)$ excitation pulse and a $180^{\circ}$ pulse (43) are shown in Fig. 7. Considering the case of the $90^{\circ}$ pulse, known as a BIR-1 pulse, the $\mathbf{B}_{1 e}$-field is initially in the transverse plane, for example, initially aligned along the $x^{\prime}$-axis (cf. Fig. 7(A)), and is rotated to the longitudinal axis. Provided that the adiabatic condition is satisfied the magnetization initially along the $z^{\prime}$-axis will remain in a plane perpendicular to the $\mathbf{B}_{1 e}$-field but will fan out due to the effects of inhomogeneities in the $\mathbf{B}_{1}$-field (cf. Fig. 7(B)). Once the $\mathbf{B}_{1 e}$-field is aligned with the $z^{\prime}$-axis it undergoes instantaneous reversal to the $-z^{\prime}$-axis (cf. Fig. 7(C)). The $\mathbf{B}_{1 e}$-field orientation is then returned to the transverse plane. For a $90^{\circ}$ pulse it should be returned to being parallel or antiparallel to the $y^{\prime}$-axis (cf. Fig. 7(D)); for a $180^{\circ}$ pulse it should be returned to the $x^{\prime}$-axis (cf. Fig. 7(E)). The effect of instantaneous reversal is to reverse the effect of $\mathbf{B}_{1}$-inhomogeneities in a similar fashion to the way in which the refocusing pulse in a spin-echo experiment reverses the effect of $\mathbf{B}_{0}$-inhomogeneities. Provided that the duration of the two halves of the pulse is equal, and the locus of the sweep of the $\mathbf{B}_{1 e}$-field during the second half of the pulse has the same form (but not necessarily the same phase) as during the first half of the pulse, then the effects of
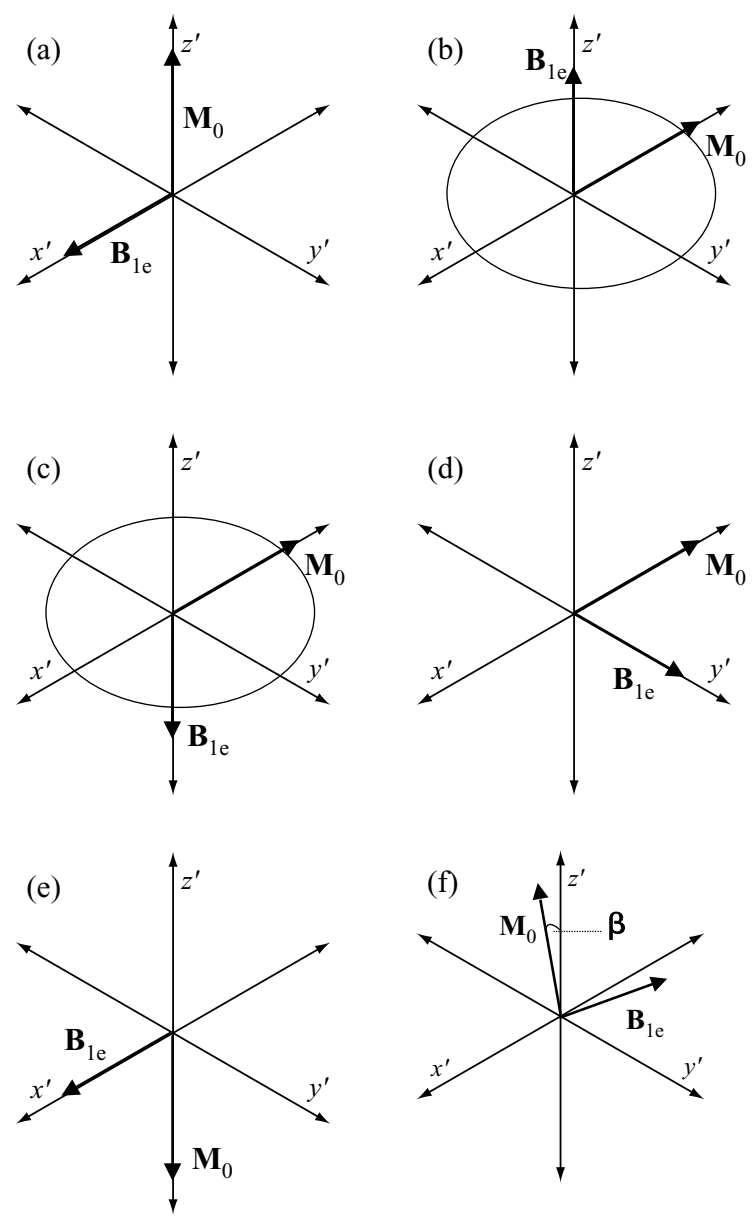

Figure 7 The BIR-1 pulse scheme. (a) shows the initial position of the magnetization vector $\mathbf{M}_{0}$ and the $\mathbf{B}_{1 e}$-field. It the time period between (a) and (b) $\mathbf{B}_{1 e}$ is swept from the $x$ - to the $z^{\prime}$-axis, and the magnetization dephases in the transverse plane due to the combined effects of inhomgeneity in $\mathbf{B}_{0}$ and $\mathbf{B}_{1}$. The orientation of $\mathbf{B}_{1 e}$ is then reversed to the $-z^{\prime}$-axis as shown in (c). For a $90^{\circ}$ excitation the $\mathbf{B}_{1 e}$-field is swept to the $y^{\prime}$-axis as shown in (d), for a $180^{\circ}$ pulse it is swept back to the $x^{\prime}$-axis as shown in (e), and for an arbitrary pulse angle it is swept back at an angle $\beta$ to the $-x^{\prime}$-axis (f).

$\mathbf{B}_{1}$-inhomogeneities will be exactly compensated. The pulse excitation angle is determined solely by the angle subtended by the initial and final orientations of the $\mathbf{B}_{1 e}$-field, and it is hence possible to program arbitrary excitation angles, independent of $\mathbf{B}_{1}$-field strength (45), as shown in Fig. 7(F). As a general remark, it should be noted that instantaneous reversal of the $\mathbf{B}_{1 e}$-field orientation can take place along any arbitrary axis. For future use it is valuable to define a concise notation which describes the sweep patterns of this type of pulse. The pulse shown in Fig. 7 can be described 
as $\left(P_{\beta}\right)^{-1} P_{0}$, a convention that was first followed in the design of composite pulses $(46,47) . P_{\beta}$ denotes a rotation of $90^{\circ}$ about an axis at an angle $\beta$ in the transverse plane as shown in Fig. 7(F). The inverse sign denotes a sweep from the transverse to the longitudinal axis.

The main weakness of the pulse schemes shown in Fig. 7 is that off-resonance effects are not compensated: off-resonance there will be a constant component of the $\mathbf{B}_{1 e}$-field along the $z^{\prime}$-axis that will not be reversed midway through the pulse. These pulses hence have an intrinsically narrow bandwidth. At the cost of increasing the pulse-duration it is possible to reduce this effect if the resonance offset of the adiabatic pulse never changes sign: one such scheme is $\left(P_{180-\beta}\right)^{-1} P_{0}\left(P_{180}\right)^{-1} P_{0}$, known as BIR-2, in which the instantaneous reversal of the $\mathbf{B}_{1 e}$-field takes place in the transverse plane. However, even with this pulse there is a deleterious effect offresonance, as the constant frequency offset will tip the $\mathbf{B}_{1 e}$-field away from the transverse plane, with the result that after the reversal in the orientation of this field the plane in which the magnetization vectors will lie will no longer be perpendicular to $\mathbf{B}_{1 e}$.

A number of adiabatic pulse schemes of this general nature have been proposed, and the most important are listed in Table 1 (43, 44, 48-50). Of these pulses, that which has gained the most widespread acceptance is that known as BIR-4 which may, at the simplest level, be viewed as a concatenation of two BIR-1 pulses. By doubling the length of the pulse it is possible to compensate for the off-resonance effects to which BIR-1 is so sensitive by ensuring that the effect of the off-resonance effects is equal and opposite between the two halves of the pulse. The total pulse angle may be arrived at by modifying the trajectory of the $\mathbf{B}_{1 e}$-field after either or

Table 1 The Major $B_{1}$ Insensitive Pulse Schemes ${ }^{a}$

\begin{tabular}{lcl}
\hline Pulse Name & Reference & \multicolumn{1}{c}{ Scheme } \\
\hline BIR-1 & 44 & $\left(P_{\beta}\right)^{-1} P_{0}$ \\
BIR-2 & 44 & $\left(P_{180-\beta}\right)^{-1} P_{0}\left(P_{180}\right)^{-1} P_{0}$ \\
BIREF-1 & 48 & $P_{0}\left(P_{180}\right)^{-1}$ \\
BIREF-2a & 48 & $\left(P_{180}\right)^{-1} P_{0}$ \\
BIR-4 & 49,50 & $\left(P_{180}\right)^{-1} P_{180+\frac{\beta}{2}}\left(P_{\frac{\beta}{2}}\right)^{-1} P_{0}$ \\
\hline
\end{tabular}

${ }^{a}$ The pulses described in Ref. 48 are exclusively refocusing pulses; the BIR pulses can be used to produce any excitation angle. both of the phase reversals, in an analagous manner as for BIR-1 (45). There is, however, a particular advantage to doing this in a symmetrical fashion (50), so that the pulse train is written as $\left(P_{180}\right)^{-1} P_{180+(\beta / 2)}\left(P_{\beta / 2}\right)^{-1} P_{0}$, to give an excitation angle of $\beta$, as the pulse then belongs to a class of pulses having reflection symmetry in time. Similar pulse trains have been examined in the context of hard composite pulses that compensate for the effects of RF inhomogeneity $(46,47)$ where it was found that only pulses with a reflection symmetry in time could combine an insensitivity to RF inhomogeneity with a broad excitation bandwidth. Although the bandwidth of BIR-4 is acceptably broad, the edges of the profile are not sufficiently sharp to make it useable for slice selective excitation.

\section{Slice Selective Pulses}

There have been several attempts to combine the desirable properties of adiabatic pulses with slice selection using magnetic field gradients. The first of these is based on using the properties of the sech pulse and trying to compensate the undesirable phase dispersion caused by off-resonance effects. The first proposal (51) is to form a composite pulse consisting of three successive sech pulses in which the duration of the first two pulses is half that of the last. The first two pulses give a rotation of $360^{\circ}$ with a phase error that is exactly opposite that generated by the last $180^{\circ}$ pulse. The compensation effect is achieved by instantaneously reversing the value of the initial frequency offset between the second and third pulses. While indeed producing an adiabatic refocusing pulse the power required for this pulse is prohibitive for in vivo applications.

A second proposal along similar lines is to only use the even echoes in a spin echo experiment in which sech pulses are used for refocusing (52). This has lower power than the previously mentioned composite pulse but represents a pulse sequence rather than a single RF pulse. The power deposition may be further reduced by simultaneously modulating both the gradient and the RF fields using the VERSE technique (53).

A second group of methods is based on using the properties of BIR-4. The first of these is termed GMAX (for gradient modulated $a$ diabatic excitation) (54). The principle here is to give the magnetic field gradient the same temporal modulation as the frequency offset of an adiabatic excitation pulse. There will be a node in this pulse 
Table 2 BISS-8 Pulse Scheme for the $B_{1}(t), \Delta \omega(t)$, and $G(t)$ Fields ${ }^{a}$

\begin{tabular}{|c|c|c|c|c|c|c|c|c|}
\hline & \multicolumn{8}{|c|}{ Period } \\
\hline & 1 & 2 & 3 & 4 & 5 & 6 & 7 & 8 \\
\hline $\mathbf{B}_{1}(t)$ & $+\Rightarrow 0$ & $0 \Rightarrow+$ & $+\Rightarrow 0$ & $0 \Rightarrow+$ & $+\Rightarrow 0$ & $0 \Rightarrow+$ & $+\Rightarrow 0$ & $0 \Rightarrow+$ \\
\hline$\Delta \omega(t)$ & $0 \Rightarrow+$ & $-\Rightarrow 0$ & $0 \Rightarrow-$ & $+\Rightarrow 0$ & $0 \Rightarrow-$ & $+\Rightarrow 0$ & $0 \Rightarrow+$ & $-\Rightarrow 0$ \\
\hline $\mathbf{G}(t)$ & $0 \Rightarrow+$ & $-\Rightarrow 0$ & $0 \Rightarrow+$ & $-\Rightarrow 0$ & $0 \Rightarrow+$ & $-\Rightarrow 0$ & $0 \Rightarrow+$ & $-\Rightarrow 0$ \\
\hline
\end{tabular}

${ }^{a}$ The notation shows the direction of the modulation for the field in question; for example, $-\Rightarrow 0$ denotes a value which is initially negative and is brought to zero.

at the point where the maximum offset induced by the gradient field is cancelled exactly by the frequency offset of the pulse: this effect is the same as that discussed previously for the use of the half-secant pulse, which is shown in Fig. 6. This node is the center of a relatively sharp transition region across which the polarity of the transverse magnetisation is reversed. The excitation is then performed a second time with the polarity of the $\mathbf{B}_{1}$-field reversed and the position of the node shifted by modifying the gradient strength and/or the amplitude of frequency modulation of the pulse. The positions of the two nodes delimit the extent of the selected slice obtained after addition of the two signals. Unfortunately this process is a two step experiment which makes it vulnerable to motion and other instabilities; furthermore the magnetization outside the excited slice is perturbed and multislice experiments are hence impractical.

In the BISS-8 experiment (55) two BIR-4 pulses are combined, with one of the pulses sandwiched between the two halves of the other, as shown in Table 2 . The total excitation angle $(\theta)$ is given by the sum of the phase discontinuities as follows:

$$
\begin{aligned}
& \Delta \phi_{1}=-\Delta \phi_{4}=180^{\circ}+\frac{\theta}{4} \\
& \Delta \phi_{2}=-\Delta \phi_{3}=-180^{\circ}+\frac{\theta}{4}
\end{aligned}
$$

The gradient modulation function during the third to sixth periods (i.e., the central pulse of the two) is the inverse of the frequency modulation function, and so in regions where the gradient field dominates the frequency modulation the two pulses produce equal and opposite excitations. The excitation angle outside the region where the frequency offset of the pulse is greater than that induced by the gradient field is hence zero. It was subsequently noted that a slice selective excitation could be generated using a single BIR-4 pulse $(56,57)$. In the mASSESS pulse of Shen and Rothman (56) and the SLAB-4 of Hsu et al.
(57), a BIR-4 pulse is played out in which during the first half of the pulse the gradient and frequency modulations have the same polarity, and in the second half of the pulse they have opposite polarity: in the regions where the frequency offset always dominates the magnetization experiences a standard BIR-4 pulse, whereas in those regions where the gradient induced offset dominates for one half of the pulse the excitation angle will be zero. This scheme is given in Table 3. The main practical problem encountered with all these pulses is the high slew-rate requirement for the magnetic field gradients, and the need to instantaneously switch the polarity of the gradients midway through the pulse. The ASSESS pulse of Shen and Rothman (56) has a smoothly varying gradient modulation function of constant polarity: this means that during the first and third sections of BIR-4 the amplitude and frequency modulations are in antiphase. This pulse generates a satisfactory in-plane excitation and an out of plane saturation, which makes it potentially of interest for spectroscopy applications but unsuitable for multislice imaging. The scheme of this pulse is given in Table 4.

In conclusion, the lack of a general formalism for generating adiabatic pulses means that slice selective pulses have only been generated by vectorial arguments. None of the pulses so far proposed is ideal in its performance, the gradient requirements in particular being taxing for in vivo applications especially on whole-body systems.

Table 3 Pulse Scheme for the mASSESS/SLAB-4

\begin{tabular}{|c|c|c|c|c|}
\hline & \multicolumn{4}{|c|}{ Period } \\
\hline & 1 & 2 & 3 & 4 \\
\hline $\mathbf{B}_{1}(t)$ & $+\Rightarrow 0$ & $0 \Rightarrow+$ & $+\Rightarrow 0$ & $0 \Rightarrow+$ \\
\hline$\Delta \omega(t)$ & $0 \Rightarrow+$ & $-\Rightarrow 0$ & $0 \Rightarrow+$ & $-\Rightarrow 0$ \\
\hline $\mathbf{G}(t)$ & $0 \Rightarrow+$ & $-\Rightarrow 0$ & $0 \Rightarrow-$ & $+\Rightarrow 0$ \\
\hline
\end{tabular}
Scheme Using the Same Notation as in Table 2 
Table 4 Pulse Scheme for the ASSESS Scheme Using the Same Notation as in Table 2

\begin{tabular}{lcccc}
\hline & \multicolumn{4}{c}{ Period } \\
\cline { 2 - 5 } & \multicolumn{1}{c}{2} & \multicolumn{1}{c}{3} & 4 \\
\hline $\mathbf{B}_{1}(t)$ & $+\Rightarrow 0$ & $0 \Rightarrow+$ & $+\Rightarrow 0$ & $0 \Rightarrow+$ \\
$\Delta \omega(t)$ & $0 \Rightarrow-$ & $+\Rightarrow 0$ & $0 \Rightarrow-$ & $+\Rightarrow 0$ \\
$\mathbf{G}(t)$ & $0 \Rightarrow+$ & $+\Rightarrow 0$ & $0 \Rightarrow+$ & $+\Rightarrow 0$ \\
\hline
\end{tabular}

\section{ACKNOWLEDGMENT}

The author thanks Thies Jochimsen for assistance in producing diagrams 3 and 4.

\section{REFERENCES}

1. Abragam A. The principles of nuclear magnetism. Oxford: Clarendon Press; 1961.

2. Le Roux P. Exact synthesis of radiofrequency waveforms. In: Proceedings of the 7th Meeting of the Society of Magnetic Resonance in Medicine, San Fransisco, 1988; p. 1049.

3. Le Roux P. Simplified rf synthesis. In: Proceedings of the 8th Meeting of the Society of Magnetic Resonance in Medicine, Amsterdam, Works in Progress 1989; p. 1168.

4. Shinnar M, Bolinger L, Leigh JS. The use of finite impulse response filters in pulse design. Magn Reson Med 1989; 12:81-87.

5. Shinnar M, Bolinger L, Leigh JS. The synthesis of soft pulses with a specified frequency response. Magn Reson Med 1989; 12:88-92.

6. Shinnar M, Eleff S, Subramanian H, Leigh JS. The synthesis of pulse sequences yielding arbitrary magnetization vectors. Magn Reson Med 1989; 12:74-80.

7. Shinnar M, Leigh JS. The application of spinors to pulse synthesis and analysis. Magn Reson Med 1989; 12:93-98.

8. Pauly J, Le Roux P, Nishimura D, Macovski A. Parameter relations for the Shinnar-Le Roux selective excitation pulse design algorithm. IEEE Trans Med Imag 1991; 10:53-65.

9. Tannús A, Garwood M. Adiabatic pulses. NMR Biomed 1997; 10:423-434.

10. Edelstein WA, Hutchison JMS, Johnson G, Redpath TW. Spin warp NMR imaging and applications to human whole-body imaging. Phys Med Biol 1980; 25:751-756.

11. Dixon WT, Du LN, Faul DD, Gado M, Rossnick S. Projection angiograms of blood labeled by adiabatic fast passage. Magn Reson Med 1986; 3:454-462.

12. Williams DS, Detre JA, Leigh JS, Koretsky AP. Magnetic resonance imaging of perfusion using spin inversion of arterial water. Proc Natl Acad Sci USA 1992; 89:212-216.
13. Zhang W, Williams DS, Koretsky AP. Measurement of rat brain perfusion by NMR using spin labeling of arterial water: in vivo determination of the degree of spin labeling. Magn Reson Med 1993; 29:416-421.

14. Talagala SL, Noll DC. Functional MRI using steady-state arterial water labeling. Magn Reson Med 1998; 39:179-183.

15. Zaharchuk G, Ledden PJ, Kwong KK, Reese TG, Rosen BR. Multislice perfusion and perfusion territory imaging in humans with separate label and image coils. Magn Reson Med 1999; 41:1093-1098.

16. Maccotta L, Detre JA, Alsop DC. The efficiency of adiabatic inversion for perfusion imaging by arterial spin labeling. NMR Biomed 1997; 10:216-221.

17. Marro KI, Hayes CE, Kushmerick MJ. A model of the inversion process in an arterial inversion experiment. NMR Biomed 1997; 10:324-332.

18. Bendall MR, Pegg DT. Uniform sample excitation with surface coils for in vivo spectroscopy by adiabatic rapid half passage. J Magn Reson 1986; 67:376-381.

19. Baum J, Tycko R, Pines A. Broadband and adiabatic inversion of a two-level system by phasemodulated pulses. Phys Rev A 1985; 32:3435-3447.

20. Hardy CJ, Edelstein WA, Vatis D, Harms R, Adams WJ. Calculated $T_{1}$ images derived from a partial saturation-inversion recovery pulse sequence with adiabatic fast passage. Magn Reson Imag 1985; 3:107-116.

21. Hardy CJ, Edelstein WA, Vatis D. Efficient adiabatic fast passage for NMR population inversion in the presence of radiofrequency field inhomgeneity and frequency offsets. J Magn Reson 1986; 66:470-482.

22. Uğurbil K, Garwood M, Rath A. Optimization of modulation functions to improve sensitivity of adiabatic pulses to variations in $\mathbf{B}_{1}$ magnitude. J Magn Reson 1988; 80:448-469.

23. Town G, Rosenfeld D. Analytic solutions to adiabatic pulse modulation functions optimized for inhomogeneous $\mathbf{B}_{1}$ fields. J Magn Reson 1990; 89:170-175.

24. Silver MS, Joseph RI, Chen CN, Sank VJ, Hoult DI. Selective population inversion in NMR. Nature 1984; 310:681-683.

25. Silver MS, Joseph RI, Hoult DI. Highly selective $90^{\circ}$ and $180^{\circ}$ pulse generation. J Magn Reson 1984; 59:347-351.

26. Silver MS, Joseph RI, Hoult DI. Selective spin inversion in nuclear magnetic resonance and coherent optics through an exact solution of the BlochRiccati equation. Phys Rev A 1985; 31:2753-2755.

27. Conolly S, Nishimura D, Macovski A. Sweepdiagram analysis of selective adiabatic pulses. J Magn Reson 1989; 83:549-564. 
28. Lawry TJ, Karczmar GS, Weiner MW, Matson GB. Computer simulation of MRS localization techniques: An analysis of ISIS. Magn Reson Med 1989; 9:299-314.

29. Norris DG, Lüdemann H, Leibfritz D. An analysis of the effects of short T2 values on the hyperbolicsecant pulse. J Magn Reson 1991; 92:94-101.

30. Bansal N. Off-resonance performance of adiabatic excitation pulses and their use for selective peak suppression. J Magn Reson B 1993; 102:73-76.

31. Ordidge RJ, Conelly A, Lohman JAB. Imageselected in vivo spectroscopy (ISIS): A new technique for spatially selective NMR spectroscopy. J Magn Reson 1986; 66:283-294.

32. Rosenfeld D, Zur Y. A new adiabatic inversion pulse. Magn Reson Med 1996; 36:124-136.

33. Rosenfeld D, Zur Y. Design of adiabatic selective pulses using optimal control theory. Magn Reson Med 1996; 36:401-409.

34. Rosenfeld D, Panfil SL, Zur Y. Design of adiabatic pulses for fat-suppression using analytic solutions of the Bloch equation. Magn Reson Med 1997; 37:793-801.

35. Kupče E, Freeman R. Adiabatic pulses for wideband inversion and broadband decoupling. J Magn Reson A 1995; 115:273-276.

36. Shen J. Use of amplitude and frequency transformations to generate adiabatic pulses of wide bandwidth and low RF power deposition. J Magn Reson B 1996; 112:131-140.

37. Kupče E, Freeman R. Stretched adiabatic pulses for broadband spin inversion. J Magn Reson A 1995; 117:246-256.

38. Kupče E, Freeman R. Optimized adiabatic pulses for wideband spin inversion. J Magn Reson A 1996; 118:299-303.

39. Tannús A, Garwood M. Improved performance of frequency-swept pulses using offset-independent adiabaticity. J Magn Reson A 1996; 120:133-137.

40. Lunati E, Cofrancesco P, Villa M, Marzola P, Sbarbati A. Evolution strategy optimization for adiabatic pulses in MRI. J Magn Reson 1999; 138:48-53.

41. Lunati E, Cofrancesco P, Villa M. New adiabatic inversion pulses for magnetic resonance imaging. Phys Med Biol 1999; 44:N99-105.

42. Ordidge R, Wylezinska M, Hugg J, Butterworth E, Franconi F. Frequency offset corrected inversion (FOCI) pulses for use in localized spectroscopy. Magn Reson Med 1996; 36:562-566.

43. Bendall MR, Garwood M, Uğurbil K, Pegg D. Adiabatic refocusing pulse which compensates for variable RF power and off-resonance effects. Magn Reson Med 1987; 4:493-499.

44. Uğurbil K, Garwood M, Bendall M. Amplitudeand frequency-modulated pulses to achieve 90 degrees plane rotations with inhomogeneous $\mathbf{B}_{1}$ fields. J Magn Reson 1987; 72:177-185.
45. Norris DG, Haase A. Variable excitation angle AFP pulses. Magn Reson Med 1989; 9:435-440.

46. Levitt M, Freeman R. NMR population inversion using a composite pulse. J Magn Reson 1979; 33:473-476.

47. Levitt M, Ernst R. Composite pulses constructed by recursive expansion procedure. J Magn Reson 1983; 55:247-253.

48. Uğurbil K, Garwood M, Rath AR, Bendall MR. Amplitude- and frequency/phase-modulated refocusing pulses that induce plane rotations even in the presence of inhomogeneous $\mathbf{B}_{1}$ fields. J Magn Reson 1988; 78:472-497.

49. Staewen RS, Johnson AJ, Ross BD, Parrish T, Merkle H, Garwood M. 3-D FLASH imaging using a single surface coil and a new adiabatic pulse, BIR-4. Invest Radiol 1990; 25:559-567.

50. Garwood M, Ke Y. Symmetric pulses to induce arbitrary flip angles with compensation for RF inhomogeneity and resonance offsets. J Magn Reson 1991; 94:511-525.

51. Conolly S, Nishimura D, Macovski A. A selective adiabatic spin-echo pulse. J Magn Reson 1989; 83:324-334.

52. Conolly S, Glover G, Nishimura D, Macovski A. A reduced power selective adiabatic spin-echo pulse sequence. Magn Reson Med 1991; 18:28-38.

53. Conolly S, Nishimura D, Macovski A, Glover G. Variable-rate selective excitation. J Magn Reson 1988; 78:440-458.

54. Johnson AJ, Garwood M, Uğurbil K. Slice selection with gradient-modulated adiabatic excitation despite the presence of large $\mathbf{B}_{1}$ inhomogeneities. J Magn Reson 1989; 81:653-660.

55. De Graaf RA, Nicolay K, Garwood M. Singleshot, $\mathbf{B}_{1}$-insensitive slice selection with a gradientmodulated adiabatic pulse, BISS-8. Magn Reson Med 1996; 35:652-657.

56. Shen J, Rothman DL. Adiabatic slice-selective excitation for surface coils. J Magn Reson 1997; 124:72-79.

57. Hsu EW, Reeder SB, MacFall JR. Single-shot, variable flip-angle slice-selective excitation with four gradient-moduled adiabatic half-passage segments. Magn Reson Med 1998; 40:334-340.

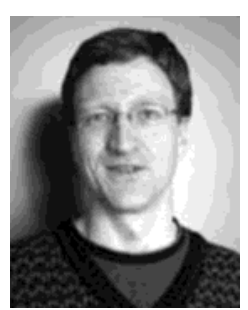

David G. Norris received his first degree in Physics from Cambridge University and subsequently obtained an M.Sc. and a Ph.D. from the University of Aberdeen. His doctoral thesis examined the subject of NMR flow imaging. He subsequently worked for eight years at the University of Bremen concentrating primarily on the themes of fast imaging and diffusion, before taking an appointment as head of the NMR group at the newly founded Max-Planck-Institute for Cognitive Neuroscience in Leipzig. Here he developed further interests in functional MRI and perfusion imaging. He is now a Principal Investigator and head of NMR at the FC Donders Centre for Cognitive Neuroimaging in Nijmegen. 